PROCEEDINGS OF THE

AMERICAN MATHEMATICAL SOCIETY

Volume 130, Number 5, Pages 1459-1466

S 0002-9939(01)06213-X

Article electronically published on October 17, 2001

\title{
MINIMAL LAGRANGIAN SUBMANIFOLDS WITH CONSTANT SECTIONAL CURVATURE IN INDEFINITE COMPLEX SPACE FORMS
}

\author{
LUC VRANCKEN
}

(Communicated by Christopher Croke)

\begin{abstract}
We study minimal Lagrangian immersions from an indefinite real space form $M_{s}^{n}(c)$ into an indefinite complex space form $\tilde{\mathbb{M}}_{s}^{n}(4 \tilde{c})$. Provided that $c \neq \tilde{c}$, we show that $M_{s}^{n}(c)$ has to be flat and we obtain an explicit description of the immersion. In the case when the metric is positive definite or Lorentzian, this result was respectively obtained by Ejiri (1982) and by Kriele and the author (1999). In the case that $c=\tilde{c}$, this theorem is no longer true; see for instance the examples discovered by Chen and the author (accepted for publication in the Tôhoku Mathematical Journal).
\end{abstract}

\section{Introduction}

Whereas Lagrangian submanifolds of positive definite complex space forms are widely studied (see a.o. [6] and the references contained therein), not much is known about Lagrangian submanifolds of indefinite complex space forms. As far as we know most results about submanifolds of indefinite complex space forms (see for example [1, 8, [9, 10] and [11]) deal with complex submanifolds. In this paper we study minimal Lagrangian submanifolds $M_{s}^{n}(c)$ with constant sectional curvature of indefinite complex space forms $\tilde{\mathbb{M}}_{s}^{n}(4 \tilde{c})$. Provided $c \neq \tilde{c}$, we obtain a complete classification and show amongst others that $M$ has to be flat. The corresponding theorems for Lagrangian immersions in Riemannian or Lorentzian complex space forms were obtained respectively by Ejiri in [4] and Kriele and the author in [5].

The paper is organized as follows. In Section 2 we recall the basic models of indefinite complex space forms and we give the basic formulas for Lagrangian submanifolds. In particular, we notice that the basic formulas are similar to those of affine hyperspheres with constant sectional curvature. This similarity allows us to apply the results of [12] to obtain an intrinsic characterization of these submanifolds and a special local frame. In Section 3, we start by recalling a result of Reckziegel 7] which allows us, using the Hopf fibration, to consider the horizontal lifts of these immersions into indefinite real space forms. We also present some examples of flat indefinite Lagrangian submanifolds. We then apply a coordinate change, motivated

Received by the editors October 7, 1999 and, in revised form, November 10, 2000.

2000 Mathematics Subject Classification. Primary 53B25, 53B30.

Key words and phrases. Lagrangian, constant sectional curvature, indefinite complex space forms.

This work was partially supported by a research fellowship of the Alexander von Humboldt Stiftung (Germany).

(C)2001 American Mathematical Society 
by the explicit form of these examples. Using these new coordinates, together with Reckziegel's result, the classification follows easily. The main difference with the approach of [4] and [5] is the use of the uniqueness part of the solution to a linear algebra problem obtained in [12] together with the explicit form of an example in order to change the coordinates before integrating.

\section{INDEFINITE COMPLEX SPACE FORMS AND THEIR LAGRANGIAN SUBMANIFOLDS}

In this section, we briefly recall some facts about indefinite complex space forms. For more details, we refer the reader to 2 . Let $\tilde{\mathbb{M}}_{s}^{n}(4 \tilde{c})$ be an indefinite complex space form of complex dimension $n$ and complex index $s$. The complex index is defined as the (complex) dimension of the largest complex negative definite vector subspace of the tangent space. The curvature tensor $\tilde{R}$ of $\tilde{\mathbb{M}}_{s}^{n}(4 \tilde{c})$ is given by

$\tilde{R}(X, Y) Z=\tilde{c}(\langle Y, Z\rangle X-\langle X, Z\rangle Y+\langle J Y, Z\rangle J X-\langle J X, Z\rangle J Y+2\langle X, J Y\rangle J Z)$,

where $J$ denotes the complex structure. We refer to [2] for the construction of the standard models of indefinite complex space forms $\mathbb{C} P_{s}^{n}(4 \tilde{c})$, when $\tilde{c}>0, \mathbb{C} H_{s}^{n}(4 \tilde{c})$, when $\tilde{c}<0$ and $\mathbb{C}_{s}^{n}$. For our purposes it is sufficient to know that there exist pseudo-Riemannian submersions, called Hopf fibrations,

$$
\Pi: S_{2 s}^{2 n+1}(\tilde{c}) \rightarrow \mathbb{C} P_{s}^{n}(4 \tilde{c}): z \mapsto z \cdot \mathbb{C}^{\star}
$$

if $\tilde{c}>0$ and if $\tilde{c}<0$ by

$$
\Pi: H_{2 s+1}^{2 n+1}(\tilde{c}) \rightarrow \mathbb{C} H_{s}^{n}(4 \tilde{c}): z \mapsto z \cdot \mathbb{C}^{\star},
$$

where

$$
\begin{aligned}
& S_{2 s}^{2 n+1}(\tilde{c})=\left\{z \in \mathbb{C}^{n+1} \mid b_{s, n+1}(z, z)=\frac{1}{\tilde{c}}\right\}, \\
& H_{2 s+1}^{2 n+1}(\tilde{c})=\left\{z \in \mathbb{C}^{n+1} \mid b_{s+1, n+1}(z, z)=\frac{1}{\tilde{c}}\right\}
\end{aligned}
$$

and $b_{p, q}$ is the standard Hermitian form with index $p$ on $\mathbb{C}^{q}$. For our convenience, we will assume that we have chosen an orthonormal basis such that the first $p$ odd terms appear with a minus sign.

In [2] it is shown that locally any indefinite complex space form is holomorphically isometric to either $\mathbb{C}_{s}^{n}, \mathbb{C} P_{s}^{n}(4 \tilde{c})$, or $\mathbb{C} H_{s}^{n}(4 \tilde{c})$. We remark that, by replacing the metric $\langle.,$.$\rangle by -\langle.,$.$\rangle , we have that \mathbb{C} H_{s}^{n}(4 \tilde{c})$ is holomorphically anti-isometric with $\mathbb{C} P_{n-s}^{n}(-4 \tilde{c})$. For that purpose, we may assume that $n-2 s \geq 0$ and if $n-2 s=0$, we only need to consider $\mathbb{C}_{s}^{n}$ and $\mathbb{C} P_{s}^{n}(4 \tilde{c})$.

Next, we consider Lagrangian submanifolds. A submanifold $M$ of a Kähler manifold is Lagrangian if and only if $J$ interchanges the tangent and the normal space. Hence a Lagrangian submanifold of an indefinite complex space form of index $s$ has real index $s$. From now on let $\tilde{\mathbb{M}}_{s}^{n}(4 \tilde{c})$ be an indefinite complex space form of holomorphic sectional curvature $4 \tilde{c}$. We denote by $\tilde{\nabla}$ the Levi Civita connection of the metric $\langle.,$.$\rangle on \tilde{\mathbb{M}}_{s}^{n}(4 \tilde{c})$. Then, the formulas of Gauss and Weingarten are respectively given by

$$
\begin{aligned}
& \tilde{\nabla}_{X} Y=\nabla_{X} Y+h(X, Y), \\
& \tilde{\nabla}_{X} J Y=-A_{J Y} X+\nabla_{X}^{\perp} J Y,
\end{aligned}
$$


defining the induced connection $\nabla$, the second fundamental form $h$, the Weingarten operator $A$ and the normal connection $\nabla^{\perp}$. Since $J$ is parallel, we deduce as in the Riemannian case that

$$
\begin{aligned}
& \nabla_{X}^{\perp} J Y=J \nabla_{X} Y, \\
& A_{J Y} X=-J h(X, Y) .
\end{aligned}
$$

We now introduce a tensor $K$ on $M$ by $K(X, Y)=K_{X} Y=-J h(X, Y)=A_{J Y} X$. It follows from the above equations that $\langle K(X, Y), Z\rangle$ is totally symmetric. A straightforward computation shows that the equations of Gauss, Codazzi and Ricci for Lagrangian submanifolds are equivalent to

$$
\begin{aligned}
& R(X, Y) Z=\tilde{c}(\langle Y, Z\rangle X-\langle X, Z\rangle Y)+\left[K_{X}, K_{Y}\right] Z, \\
& \left(\nabla_{X} K\right)(Y, Z)=\left(\nabla_{Y} K\right)(X, Z) .
\end{aligned}
$$

A Lagrangian submanifold $M_{s}^{n}$ with constant sectional curvature $c$ is minimal if and only if

$$
\operatorname{trace} K_{X}=0
$$

for every tangent vector field $X$. The Gauss and Codazzi equations reduce to

$$
\begin{aligned}
& {\left[K_{X}, K_{Y}\right] Z=a(\langle Y, Z\rangle X-\langle X, Z\rangle Y),} \\
& \left(\nabla_{X} K\right)(Y, Z)=\left(\nabla_{Y} K\right)(X, Z),
\end{aligned}
$$

where $a=c-\tilde{c}$.

Remark that, since $\nabla$ is the Levi Civita connection of the metric $\langle.,$.$\rangle , this$ is exactly the situation which also appears in affine differential geometry when one investigates affine hyperspheres with constant sectional curvature; see [12. Therefore, recalling the results of [12], we obtain in particular the following:

Theorem 2.1. Let a be a nonzero number, let $n$ be the dimension and let $s$ be a nonnegative integer number with $n-2 s \geq 0$. Then:

(i) If $n-2 s>1$ and $a$ is negative, then there does not exist a metric $\langle.,$.$\rangle (with$ signature $s$ ) and a tensor $K$, which is totally symmetric with respect to $\langle.,$.$\rangle ,$ on $\mathbb{R}^{n}$ which satisfy (2.3) and (2.4).

(ii) Otherwise, solutions $\langle.,$.$\rangle (with index s$ ) and $K$ do exist. Moreover any other solution $(\tilde{h}, \tilde{K})$ is obtained by

$$
\begin{aligned}
& \tilde{h}(x, y)=\langle A x, A y\rangle, \\
& \tilde{K}(x, y)=A^{-1} K(A x, A y),
\end{aligned}
$$

where $A$ is an arbitrary linear automorphism of $\mathbb{R}^{n}$.

Theorem 2.2. Let $\phi: M_{s}^{n}(c) \rightarrow \tilde{M}_{s}^{n}(4 \tilde{c})$ be a minimal Lagrangian isometric immersion. Suppose that $n-2 s \geq 0$ and that $c \neq \tilde{c}$. Then, if $n-2 s>1$, we must have that $c<\tilde{c}$. Moreover, let $p \in M_{s}^{n}(c)$. Then there exist vector fields $\left\{U_{1}, V_{1}, \ldots, U_{s}, V_{s}, E_{1}, \ldots, E_{r}\right\}$, defined on a neighborhood of $p$, where $r=n-2 s$ such that $\left\langle E_{k}, E_{\ell}\right\rangle=\delta_{k \ell},\left\langle U_{m}, V_{j}\right\rangle=\delta_{m j}$, where $j, m=1, \ldots, s$ and $k, \ell=1, \ldots, r$ and all other components of the metric vanish. Assuming moreover that $\ell<k$ and 
$m<j$, we have that $K$ is given by

$U_{j}, V_{j}$ and $E_{k}$ are eigenvectors of $K_{U_{m}}$ with eigenvalue $\lambda_{m}$,

$U_{j}, V_{j}$ and $E_{k}$ are eigenvectors of $K_{V_{m}}$ with eigenvalue $\frac{(n-2 m+2)}{2} \lambda_{m}^{2}$,

$$
\begin{aligned}
& K\left(U_{j}, U_{j}\right)=-\frac{n-2 j}{2} \lambda_{j} U_{j}+V_{j}, \\
& K\left(U_{j}, V_{j}\right)=\sum_{m=1}^{j-1}\left(\lambda_{m} V_{m}+\frac{(n-2 m+2)}{2} \lambda_{m}^{2} U_{m}\right)-\frac{(n-2 j)(n-2 j+2)}{4} \lambda_{j}^{2} U_{j}-\frac{(n-2 j)}{2} \lambda_{j} V_{j}, \\
& K\left(V_{j}, V_{j}\right)=\frac{(n-2 j+2)^{3}}{8} \lambda_{j}^{3} U_{j}-\frac{1}{4}(n-2 j)(n-2 j+2) \lambda_{j}^{2} V_{j}, \\
& K\left(E_{k}, E_{\ell}\right)=-\frac{\mu_{\ell}}{r-\ell} E_{k}, \\
& K\left(E_{k}, E_{k}\right)=\sum_{j=1}^{s}\left(\lambda_{j} V_{j}+\frac{(n-2 j+2)}{2} \lambda_{j}^{2} U_{j}\right)-\sum_{\ell=1}^{k-1} \frac{\mu_{\ell}}{r-\ell} E_{\ell}+\mu_{k} E_{k},
\end{aligned}
$$

where the $\mu_{k}$ and $\lambda_{j}$ are constants determined by

$$
\begin{aligned}
\lambda_{j}^{3} & =-\frac{2 a(n+1)}{(n-2 j+2)(n-2 j+1)(n-2 j+3)}, \\
\mu_{j}^{2} & =-\frac{a(n+1)(r-j)^{2}}{(r-j+1)(r-j+2)} .
\end{aligned}
$$

Using the fact that $K$ is a Codazzi tensor with respect to $\nabla$, (2.5), it is then straightforward; see also [12] to show the following:

Lemma 2.3. Let $\left\{U_{1}, V_{1}, \ldots, U_{s}, V_{s}, W_{1}, \ldots, W_{r}\right\}$ be the frame constructed before. Then all connection coefficients (with respect to $\nabla$ ) vanish. In particular $M$ has flat metric, i.e. $c=0$.

\section{Classification Results}

Using the results of the previous section, we already obtain the following corollaries:

Corollary 3.1. Let $M_{s}^{n}$ be a minimal Lagrangian submanifold with constant sectional curvature $c$ of $\mathbb{C} H_{s}^{n}(4 \tilde{c}), n-2 s>1$, where $\tilde{c}<0$. Then $c=\tilde{c}$.

Proof. Suppose that $c \neq \tilde{c}$. In that case, $a \neq 0$. Since $n-2 s>1$, it follows from the previous theorem (2.7) that $a$ has to be negative. Since, from the previous lemma, we know that our Lagrangian submanifold has to be flat, we find that $\tilde{c}$ is positive. This is a contradiction.

Of course, also in the flat case, a similar theorem can be obtained:

Corollary 3.2. Let $M_{s}^{n}$ be a minimal Lagrangian submanifold with constant sectional curvature $c$ of $\mathbb{C}_{s}^{n}$. Then $c=0$.

In general, applying the results of the previous chapters, a classification result can be proved. However, in order to obtain explicit equations, we first recall some basic facts from [7] which relate Lagrangian submanifolds of respectively $\mathbb{C} P_{s}^{n}(4 \tilde{c})$ and $\mathbb{C} H_{s}^{n}(4 \tilde{c})$ to horizontal immersions in respectively $S_{2 s}^{2 n+1}(\tilde{c})$ and $H_{2 s+1}^{2 n+1}(\tilde{c})$. Here a horizontal immersion $f: M_{s}^{n} \rightarrow S_{2 s}^{2 n+1}(\tilde{c})$ (respectively, $f: M_{s}^{n} \rightarrow H_{2 s+1}^{2 n+1}(\tilde{c})$ ) is an immersion which satisfies if $(p) \perp f_{*}\left(T_{p} M_{s}^{n}\right)$ for all $p \in M_{s}^{n}$, where $\mathrm{i}=\sqrt{-1}$. 
Theorem $3.3([7])$. Let $\tilde{c}>0$ and let $\Pi: S_{2 s}^{2 n+1}(\tilde{c}) \rightarrow \mathbb{C} P_{s}^{n}(4 \tilde{c})$ be the Hopf fibration. If $f: M_{s}^{n} \rightarrow S_{2 s}^{2 n+1}(\tilde{c})$ is a horizontal immersion, then $F=\Pi \circ f: M_{s}^{n} \rightarrow$ $\mathbb{C} P_{s}^{n}(4 \tilde{c})$ is a Lagrangian immersion.

Conversely, let $M_{s}^{n}$ be a simply connected manifold and let $F: M_{s}^{n} \rightarrow \mathbb{C} P_{s}^{n}(4 \tilde{c})$ be a Lagrangian immersion. Then there exist a 1-parameter family of horizontal lifts $f: M_{s}^{n} \rightarrow S_{2 s}^{2 n+1}(\tilde{c})$ such that $F=\Pi \circ f$. Any two such lifts $f_{1}$ and $f_{2}$ are related by $f_{1}=e^{i \theta} f_{2}$, where $\theta$ is a constant.

The analogous statement for $\tilde{c}<0$ also holds if one replaces $S_{2 s}^{2 n+1}(\tilde{c})$ by $H_{2 s+1}^{2 n+1}(\tilde{c})$ and $\mathbb{C} P_{s}^{n}(4 \tilde{c})$ by $\mathbb{C} H_{s}^{n}(4 \tilde{c})$. Remark that both immersions have the same induced metric and also that the second fundamental forms of both immersions are closely related. For more details see 7 .

Before continuing with the classification, we now want to use the above theorem to present two classes of indefinite flat Lagrangian submanifolds.

Example 3.4. We define an immersion $\Phi$ of $\mathbb{R}^{n}$ into $S_{2 s}^{2 n+1}(\tilde{c})$ by

$$
\begin{aligned}
\Phi\left(u_{1}, v_{1}, \ldots, u_{s}, v_{s}, w_{1}, \ldots, w_{r}\right)= & \frac{\alpha}{\sqrt{n+1}}\left(\sqrt{2} e^{i u_{1}} \sinh v_{1}, \sqrt{2} e^{i u_{1}} \cosh v_{1}, \ldots,\right. \\
& \left.\sqrt{2} e^{i u_{s}} \sinh v_{s}, \sqrt{2} e^{i u_{s}} \cosh v_{s}, e^{i w_{1}}, \ldots, e^{i w_{r}}, e^{i \beta}\right),
\end{aligned}
$$

where $\alpha^{2}=\frac{1}{\tilde{c}}, r=2 n-s$ and $\beta=-2 \sum_{j=1}^{s} u_{j}-\sum_{k=1}^{r} w_{k}$. Clearly, remembering that the first $s$ odd terms have to be taken with a minus sign, $\langle\Phi, \Phi\rangle=\alpha^{2}$. We also have that

$$
\begin{aligned}
\Phi_{u_{1}} & =\frac{\alpha}{\sqrt{n+1}}\left(i \sqrt{2} e^{i u_{1}} \sinh v_{1}, i \sqrt{2} e^{i u_{1}} \cosh v_{1}, 0, \ldots, 0,-2 i e^{i \beta}\right), \\
\Phi_{v_{1}} & =\frac{\alpha}{\sqrt{n+1}}\left(\sqrt{2} e^{i u_{1}} \cosh v_{1}, \sqrt{2} e^{i u_{1}} \sinh v_{1}, 0, \ldots, 0,0\right), \\
\Phi_{w_{1}} & =\frac{\alpha}{\sqrt{n+1}}\left(0, \ldots, 0, i e^{i w_{1}}, 0, \ldots, 0,-i e^{i \beta}\right) .
\end{aligned}
$$

From the above equations, it follows straightforwardly that $\Phi$ is horizontal and thus $\Pi \circ \Phi$ is a Lagrangian immersion in $\mathbb{C} P_{s}^{n}(4 \tilde{c})$. We now introduce a vector field $\eta$ by

$$
\eta=\frac{\alpha}{\sqrt{n+1}}\left(0, \ldots, 0, e^{i \beta}\right) .
$$

It then follows that

$$
\eta=\frac{1}{n+1}\left(\Phi+i \sum_{j=1}^{s} \Phi_{u_{j}}+i \sum_{k=1}^{r} \Phi_{w_{k}}\right) .
$$

Using this, we obtain for the second derivatives of the immersion the following formulas:

$$
\begin{array}{ll}
\Phi_{v_{j} v_{j}}=-i \Phi_{u_{j}}+2 \eta, & \Phi_{u_{j} u_{j}}=i \Phi_{u_{j}}-6 \eta, \\
\Phi_{u_{j} v_{j}}=i \Phi_{v_{j}}, & \Phi_{u_{j} u_{m}}=-4 \eta, \\
\Phi_{u_{j} w_{k}}=-2 \eta, & \Phi_{w_{k} w_{k}}=i \Phi_{w_{k}}-2 \eta, \\
\Phi_{w_{k} w_{\ell}}=-\eta, & \Phi_{v_{j} v_{m}}=0, \\
\Phi_{v_{j} w_{k}}=0, & \Phi_{v_{j} u_{m}}=0 .
\end{array}
$$

Since the above expressions do not contain any tangential terms, we obtain that all connection coefficients vanish and hence $\Phi$ (and also $\Pi \circ \Phi$ ) is flat. Using the above formulas, together with the fact that $A_{i X} Y=-i h(X, Y)$, where $h$ and $A$ 
denote respectively the second fundamental form and the Weingarten operator of the immersion in the indefinite sphere, we also get that

$$
\begin{aligned}
& \operatorname{trace} A_{i \Phi_{v_{j}}}=0, \\
& \operatorname{trace} A_{i \Phi_{u_{j}}}=1-\frac{6}{n+1}+1-\frac{4(s-1)}{n+1}-2 \frac{r}{n+1}=0, \\
& \operatorname{trace} A_{i \Phi_{w_{k}}}=-\frac{2 s}{n+1}+1-\frac{2}{n+1}-\frac{r-1}{n+1}=0,
\end{aligned}
$$

implying that the immersion is minimal in the indefinite sphere. We also obtain, using [7], that the tensor $K$ and the metric are respectively given by

$$
\begin{array}{ll}
K\left(\partial_{v_{j}}, \partial_{v_{j}}\right)=-\partial_{u_{j}}+2 \tilde{\eta}, & K\left(\partial_{u_{j}}, \partial_{u_{j}}\right)=\partial_{u_{j}}-6 \tilde{\eta}, \\
K\left(\partial_{u_{j}}, \partial_{v_{j}}\right)=\partial_{v_{j}}, & K\left(\partial_{u_{j}}, \partial_{u_{m}}\right)=-4 \tilde{\eta}, \\
K\left(\partial_{u_{j}}, \partial_{w_{k}}\right)=-2 \tilde{\eta}, & K\left(\partial_{w_{k}}, \partial_{w_{k}}\right)=\partial_{w_{k}}-2 \tilde{\eta}, \\
K\left(\partial_{w_{k}}, \partial_{w_{\ell}}\right)=-\tilde{\eta}, & K\left(\partial_{v_{j}}, \partial_{v_{m}}\right)=0, \\
K\left(\partial_{v_{j}}, \partial_{w_{k}}\right)=0, & K\left(\partial_{v_{j}}, \partial_{u_{m}}\right)=0,
\end{array}
$$

where $j \neq m$ and $k \neq \ell$ and $\tilde{\eta}$ is defined by

$$
\tilde{\eta}=\frac{1}{n+1}\left(\sum_{j=1}^{s} \partial_{u_{j}}+\sum_{k=1}^{r} \partial_{w_{k}}\right)
$$

and

$$
\begin{array}{ll}
\left\langle\partial_{v_{j}}, \partial_{v_{j}}\right\rangle=-\frac{2 \alpha^{2}}{(n+1)}, & \left\langle\partial_{u_{j}}, \partial_{u_{j}}\right\rangle=\frac{6 \alpha^{2}}{(n+1)}, \\
\left\langle\partial_{u_{j}}, \partial_{v_{j}}\right\rangle=0, & \left\langle\partial_{u_{j}}, \partial_{u_{m}}\right\rangle=\frac{4 \alpha^{2}}{(n+1)}, \\
\left\langle\partial_{u_{j}}, \partial_{w_{k}}\right\rangle=\frac{2 \alpha^{2}}{(n+1)}, & \left\langle\partial_{w_{k}}, \partial_{w_{k}}\right\rangle=\frac{2 \alpha^{2}}{(n+1)}, \\
\left\langle\partial_{w_{k}}, \partial_{w_{\ell}}\right\rangle=\frac{\alpha^{2}}{(n+1)}, & \left\langle\partial_{v_{j}}, \partial_{v_{m}}\right\rangle=0, \\
\left\langle\partial_{v_{j}}, \partial_{w_{k}}\right\rangle=0, & \left\langle\partial_{v_{j}}, \partial_{u_{m}}\right\rangle=0 .
\end{array}
$$

By a straightforward computation or by using the fact that $\Phi$ is horizontal and thus $\Pi \circ \Phi$ is Lagrangian, we see that the index of the above metric equals $s$.

Example 3.5. For the next example, we assume that $n-2 s=1$ and we define an immersion $\Psi$ of $\mathbb{R}^{n}$ into $H_{2 s}^{2 n+1}(\tilde{c})$ by

$$
\begin{aligned}
\Psi\left(u_{1}, v_{1}, \ldots, u_{s}, v_{s}, w\right)= & \frac{\alpha}{\sqrt{s+1}}\left(e^{i u_{1}} \cosh v_{1}, e^{i u_{1}} \sinh v_{1}, \ldots, e^{i u_{s}} \cosh v_{s},\right. \\
& \left.e^{i u_{s}} \sinh v_{s}, e^{-i \beta} \cosh w, e^{-i \beta} \sinh w\right),
\end{aligned}
$$

where $\alpha^{2}=-\frac{1}{\tilde{c}}$ and $\beta=\sum_{j=1}^{s} u_{j}$. Clearly, remembering that the first $s+1$ odd terms have to be taken with a minus sign, $\langle\Psi, \Psi\rangle=-\alpha^{2}$. That $\Psi$ is horizontal, minimal and flat now follows in a similar way as in the previous example.

Then, if we denote by $\mathbb{M}_{s}^{n}(4 \tilde{c})=\mathbb{C} P_{s}^{n}(4 \tilde{c})$ if $\tilde{c}>0$ and $\mathbb{M}_{s}^{n}(4 \tilde{c})=\mathbb{C} H_{s}^{n}(4 \tilde{c})$ if $\tilde{c}<0$ we have the following result:

Theorem 3.6. Let $\phi: M_{s}^{n}(c) \rightarrow \mathbb{M}_{s}^{n}(4 \tilde{c})$ be a minimal isometric Lagrangian immersion into $\mathbb{M}_{s}^{n}(4 \tilde{c}), n-2 s \geq 0$. Suppose that $c \neq \tilde{c}$. Then $c=0$, and if $n-2 s>1$, then $\tilde{c}>0$. Moreover if $\tilde{c}>0$, we get that $\phi$ is locally congruent with the immersion $\Pi \circ \Phi$, as defined in Example 3.4, and if $\tilde{c}<0$ and $n-2 s=1, \phi$ is locally congruent with the immersion $\Pi \circ \Psi$, as defined in Example 3.5. 
Proof. We already know that $c=0$. Next we assume that $n-2 s>1$ and $\tilde{c}>$ 0 . Now, let $V$ be an $n$-dimensional vector space. Then, on $V$ we can define an operator $K$ (resp. $\tilde{K}$ ) and a metric $h($ resp. $\tilde{h}$ ) as in Theorem 2.2 (resp. Example 3.4). However, by the uniqueness theorem, we know that there exists a linear transformation such that

$$
\begin{array}{r}
\tilde{K}(x, y)=A^{-1} K(A x, A y), \\
\tilde{h}(x, y)=h(A x, A y) .
\end{array}
$$

This means that the expression of $\tilde{K}$ and $\tilde{h}$ with respect to a basis $\left\{A e_{1}, \ldots, A e_{n}\right\}$ is the same as the expression of $K$ and $h$ with respect to the basis $\left\{e_{1}, \ldots, e_{n}\right\}$.

Now taking a point $p$ and the basis constructed in Theorem 2.2, we then know from the above that there exists a transformation of this basis, with a matrix $B$, such that the components of the metric and the difference tensor with respect to this new basis are as in Example 3.4. Since all components are constant, we can take the same matrix $B$ at every point. Since for the old basis all connection coefficients vanish, the same is true for the new basis. Consequently there exist coordinates $u_{1}, v_{1}, \ldots, u_{s}, v_{s}, w_{1}, \ldots, w_{k}$ on $M_{s}^{n}$ such that the Hopf lift of the immersion of $M_{s}^{n}$ into $\mathbb{M}_{s}^{n}(4 \tilde{c})$, which we denote by $x$, is characterized by the following system of differential equations:

$$
\begin{array}{ll}
x_{v_{j} v_{j}}=-i x_{u_{j}}+2 \eta, & x_{u_{j} u_{j}}=i x_{u_{j}}-6 \eta, \\
x_{u_{j} v_{j}}=i x_{v_{j}}, & x_{u_{j} u_{m}}=-4 \eta, \\
x_{u_{j} w_{k}}=-2 \eta, & x_{w_{k} w_{k}}=i x_{w_{k}}-2 \eta, \\
x_{w_{k} w_{\ell}}=-\eta, & x_{v_{j} v_{m}}=0, \\
x_{v_{j} w_{k}}=0, & x_{v_{j} u_{m}}=0,
\end{array}
$$

where $\eta=\frac{1}{n+1}\left(x+i \sum_{m=1}^{s} x_{u_{m}}+i \sum_{k=\ell}^{r} x_{w_{\ell}}\right)$. It now follows that

$$
\begin{aligned}
& \eta_{v_{j}}=\frac{1}{n+1}\left(x_{v_{j}}+i x_{u_{j} v_{j}}\right)=0, \\
& \eta_{u_{j}}=\frac{1}{n+1}(-6 i \eta-4 i(s-1) \eta-2 i r \eta)=-2 i \eta, \\
& \eta_{w_{k}}=\frac{1}{n+1}(-2 s i \eta-2 i \eta-(r-1) i \eta=-i \eta .
\end{aligned}
$$

Consequently there exists a constant vector $C_{n+1}$ such that

$$
\eta=C_{n+1} e^{-2 i \sum_{j=1}^{s} u_{j}-i \sum_{k=1}^{r} w_{k}} .
$$

The system of differential equations for $x$ can now be rewritten as

$$
\begin{array}{ll}
(x-\eta)_{v_{j} v_{j}}=-i(x-\eta)_{u_{j}}, & (x-\eta)_{u_{j} u_{j}}=i(x-\eta)_{u_{j}}, \\
(x-\eta)_{u_{j} v_{j}}=i(x-\eta)_{v_{j}}, & (x-\eta)_{u_{j} u_{m}}=0, \\
(x-\eta)_{u_{j} w_{k}}=0, & (x-\eta)_{w_{k} w_{k}}=i(x-\eta)_{w_{k}}, \\
(x-\eta)_{w_{k} w_{\ell}}=0, & (x-\eta)_{v_{j} v_{m}}=0, \\
(x-\eta)_{v_{j} w_{k}}=0, & (x-\eta)_{v_{j} u_{m}}=0 .
\end{array}
$$

It now follows that there exist constants $C_{1}, D_{1}, \ldots, C_{s}, D_{s}, C_{2 s+1}, \ldots, C_{n}$ such that

$$
x-\eta=\sum_{j=1}^{s}\left(C_{j} e^{i u_{j}} \sinh v_{j}+D_{j} e^{i u_{j}} \cosh v_{j}\right)+\sum_{k=1}^{r} C_{2 s+k} e^{i w_{k}} .
$$


Since $i x$ is normal to the immersion (horizontally) and $\langle x, x\rangle=\alpha^{2}=\frac{1}{\tilde{c}}$, it now follows that

$$
\begin{aligned}
& \left\langle C_{j}, C_{j}\right\rangle=-\left\langle D_{j}, D_{j}\right\rangle, \\
& \left\langle D_{j}, D_{j}\right\rangle-2\left\langle C_{n+1}, C_{n+1}\right\rangle=0, \\
& \left\langle C_{2 s+k}, C_{2 s+k}\right\rangle-\left\langle C_{n+1}, C_{n+1}\right\rangle=0, \\
& \sum_{j=1}^{s}\left\langle D_{j}, D_{j}\right\rangle+\sum_{k=1}^{r}\left\langle C_{2 s+k}, C_{2 s+k}\right\rangle+\left\langle C_{n+1}, C_{n+1}\right\rangle=\alpha^{2},
\end{aligned}
$$

and all other inner products of the form $\left\langle C_{.}, i D_{.}\right\rangle,\left\langle C_{.}, D_{.}\right\rangle,\left\langle C_{.}, i C_{.}\right\rangle, \ldots$ vanish. Hence $\phi$ is locally congruent with the immersion $\Phi$ described in Example 3.4. In case that $\tilde{c}<0$ and $n-2 s=1$ we proceed in a similar fashion to obtain that $\phi$ is congruent with the immersion $\Psi$ as described in Example 3.5.

Remark that although the basis described in Example 3.4 and Example 3.5 is extremely convenient for integration and obtaining the explicit formulas for the immersion, it is not particular suited to prove for example Lemma 2.3. For that purpose it is easier to use the basis mentioned in Theorem 2.2.

\section{REFERENCES}

[1] K. Abe and M. Magid. Indefinite rigidity of complex submanifolds and maximal surfaces. Math. Proc. Camb. Philos. Soc, 106:481-494, 1989. MR 90g:53075

[2] M. Barros and A. Romero. Indefinite Kähler manifolds. Math. Ann., 261:55-62, 1982. MR 84d:53033

[3] B. Y. Chen and L. Vrancken. Lagrangian minimal immersions of a lorentzian real space form $M_{1}^{n}(c)$ into a lorentzian complex space form $\tilde{M}_{1}^{n}(4 c)$, accepted for publication in Tôhoku Mathematical Journal.

[4] N. Ejiri. Totally real minimal immersions of $n$-dimensional real space forms into $n$-dimensional complex space forms. Proc. Amer. Math. Soc., 84:243-246, 1982. MR 83a:53055

[5] M. Kriele and L. Vrancken. Minimal lagrangian submanifolds of lorentzian complex space forms with constant sectional curvature. Archiv für Mathematik, 72:223-232, 1999. MR 2000a:53110

[6] J. M. Morvan. Minimal lagrangian submanifolds, a survey. In Geometry and Topology of Submanifolds, volume 3, pages 206-226, Singapore, 1991. World Scientific. MR 96d:53072

[7] H. Reckziegel. Horizontal lifts of isometric immersions into the bundle space of a pseudoRiemannian submersion. In D. Ferus, R. B. Gardner, S. Helgason, and U. Simon, editors, Global Differential Geometry and Global Analysis 1984. Proceedings of a Conference held in Berlin, June 10-14, 1984, volume 1156 of Lecture notes in Mathematics, pages 264-279. Springer-Verlag, 1984. MR 87e:53103

[8] A. Romero. On a certain class of complex Einstein hypersurfaces in indefinite complex space forms. Math. Z., 192:627-635, 1986. MR 87k:53109

[9] A. Romero. Some examples of indefinite complete complex Einstein hypersurfaces not locally symmetric. Proc. Amer. Math. Soc., 98:283-286, 1986. MR 87j:53096

[10] A. Romero. An extension of Calabi's rigidity theorem to complex submanifolds of indefinite space forms. Manuscripta Math., 59:261-276, 1987. MR 89a:53064

[11] M. Umehara. Diastasis and real analytic functions on complex manifolds. J. Math. Soc. Japan, 40:201-228, 1988. MR 89m:32019

[12] L. Vrancken. The Magid-Ryan conjecture for equiaffine hyperspheres with constant sectional curvature. Journal of Differential Geometry, 54:99-138, 2000.

Mathematisch Instituut, Universiteit Utrecht, Budapestlaan 6, 3584CD Utrecht, The Netherlands

E-mail address: vrancken@math.uu.nl 